\title{
Transcriptome reprogramming, epigenetic modifications and alternative splicing orchestrate the tomato root response to the beneficial fungus Trichoderma harzianum
}

\author{
Monica De Palma ${ }^{1}$, Maria Salzano ${ }^{1}$, Clizia Villano², Riccardo Aversano ${ }^{2}$, Matteo Lorito², Michelina Ruocco ${ }^{3}$, \\ Teresa Docimo ${ }^{1}$, Anna Lisa Piccinelli', Nunzio D'Agostino (D) ${ }^{5}$ and Marina Tucci (ID ${ }^{1}$
}

\begin{abstract}
Beneficial interactions of rhizosphere microorganisms are widely exploited for plant biofertilization and mitigation of biotic and abiotic constraints. To provide new insights into the onset of the roots-beneficial microorganisms interplay, we characterised the transcriptomes expressed in tomato roots at 24,48 and $72 \mathrm{~h}$ post inoculation with the beneficial fungus Trichoderma harzianum T22 and analysed the epigenetic and post-trascriptional regulation mechanisms. We detected 1243 tomato transcripts that were differentially expressed between Trichoderma-interacting and control roots and $83 \mathrm{~T}$. harzianum transcripts that were differentially expressed between the three experimental time points. Interaction with Trichoderma triggered a transcriptional response mainly ascribable to signal recognition and transduction, stress response, transcriptional regulation and transport. In tomato roots, salicylic acid, and not jasmonate, appears to have a prominent role in orchestrating the interplay with this beneficial strain. Differential regulation of many nutrient transporter genes indicated a strong effect on plant nutrition processes, which, together with the possible modifications in root architecture triggered by ethylene/indole-3-acetic acid signalling at $72 \mathrm{~h}$ post inoculation may concur to the well-described growth-promotion ability of this strain. Alongside, T. harzianum-induced defence priming and stress tolerance may be mediated by the induction of reactive oxygen species, detoxification and defence genes. A deeper insight into gene expression and regulation control provided first evidences for the involvement of cytosine methylation and alternative splicing mechanisms in the plant-Trichoderma interaction. A model is proposed that integrates the plant transcriptomic responses in the roots, where interaction between the plant and beneficial rhizosphere microorganisms occurs.
\end{abstract}

Correspondence: Nunzio D'Agostino (nunzio.dagostino@crea.gov.it) or Marina Tucci (mtucci@unina.it)

'Institute of Biosciences and BioResources, Research Division Portici, National Research Council, 80055 Portici, Italy

${ }^{2}$ Department of Agricultural Sciences, University of Naples Federico II, 80055 Portici, Italy

Full list of author information is available at the end of the article.

These authors contributed equally: Monica De Palma, Maria Salzano

These authors jointly supervised this work: Nunzio D'Agostino, Marina Tucci

\section{Introduction}

The need for safeguarding both human and environmental health as well as for preserving natural resources requires an effective crop management combined with a substantially reduced application of agrochemicals. To this purpose, beneficial rhizosphere microbes are being increasingly exploited, for example for biofertilization,

\section{(c) The Author(s) 2019}

(c) (i) Open Access This article is licensed under a Creative Commons Attribution 4.0 International License, which permits use, sharing, adaptation, distribution and reproduction in any medium or format, as long as you give appropriate credit to the original author(s) and the source, provide a link to the Creative Commons license, and indicate if changes were made. The images or other third party material in this article are included in the article's Creative Commons license, unless indicated otherwise in a credit line to the material. If material is not included in the article's Creative Commons license and your intended use is not permitted by statutory regulation or exceeds the permitted use, you will need to obtain permission directly from the copyright holder. To view a copy of this license, visit http://creativecommons.org/licenses/by/4.0/. 
disease and pest control, alleviation of environmental constraints $^{1,2}$. Plant growth-promoting fungi (PGPF) include many strains of Trichoderma spp., which are also able to colonise roots, behaving as opportunistic symbionts. Along with direct biocontrol of soil pathogens, Trichoderma spp. can exert stimulation of the plant immune system (induced systemic resistance, ISR), and pre-activation (priming) of molecular mechanisms of defence against a broad range of pathogens $^{3-7}$. In addition, positive effects of these PGPF on plant growth and alleviation of abiotic stresses have been widely reported $^{8-10}$.

The cascade of molecular events that characterises the onset of the plant-Trichoderma interaction has been documented by several proteomic and transcriptomic studies, mainly on the aboveground part of the plant ${ }^{11-14}$. Perception of microbe-associated molecular patterns (MAMPs) by pattern recognition receptors (PRRs) mediates early perception and activates MAMPs/DAMPstriggered immunity (MTI/DTI $)^{15,16}$. To date, only a few genes coding for receptor/recognition protein-Trichoderma elicitor pairs have been characterised ${ }^{17}$. Trichoderma effectors have also been suggested to activate effector-triggered immunity $(\mathrm{ETI})^{17}$. Indeed, Trichoderma-stimulated cell death during root colonisation ${ }^{18}$, a phenomenon that is suggestive of ETI-induced programmed cell death. Among the early events induced by host-Trichoderma recognition, both salicylic acid (SA)mediated and jasmonate (JA)/ethylene (Et)-mediated signalling have been implicated, but also abscisic acid (ABA) and indole-3-acetic acid (IAA) have been proposed to play important roles ${ }^{17,19}$. Recent studies indicate that regulatory mechanisms, such as epigenetic (e.g. DNA methylation, histone modification) and posttranscriptional (e.g. alternative splicing, AS) modifications, are key pathogenesis modulators ${ }^{20,21}$ and could also be involved in establishing beneficial interactions ${ }^{21}$. In potato, higher cytosine DNA methylation is implicated in suppression of the bacterial endophyte Burkholderia phytofirmans-induced plant growth stimulation ${ }^{22}$, suggesting that DNA (de)methylation could be relevant to beneficial interactions. Recently, evaluation of AS patterns from different plant species, including tomato, revealed that $39-70 \%$ of multi-exon genes produces at least one splice variants ${ }^{23}$ and that most genes related to plant defence undergo AS during plant-pathogen interactions $^{20,24,25}$. However, AS remains poorly studied in plants and even less in Solanaceous species ${ }^{26,27}$.

Despite the fact that recognition of rhizospherecompetent Trichoderma strains and the elicitation of plant responses are exclusively mediated by the root system $^{28-30}$, investigations on the belowground interplay between roots and beneficial fungi have so far been limited $^{8,30,31}$. In such plant organ, the few available data indicate that $A$. thaliana colonisation by $T$. asperelloides
T203 requires activation of the JA pathway and enhanced expression of specific WRKY transcription factors (TFs), which stimulate JA signalling via suppression of jasmonate ZIM domain (JAZ) repressors ${ }^{8}$. SA was suggested to be involved in limiting Trichoderma root colonisation based on results with $T$. harzianum after interaction with the SA-impaired sid2 mutant of A. thaliana ${ }^{32,33}$ or with oil palm roots ${ }^{31}$. Trichoderma also activates an efficient reactive oxygen species (ROS) detoxification system and production of antimicrobial compounds through the phenylpropanoid pathway, which may participate in limiting pathogen infection, but also in ameliorating plant tolerance to abiotic stresses ${ }^{3,8,34,35}$. Members of the antioxidant machinery activated by Trichoderma spp. include antioxidant enzymes, such as superoxide dismutase (SOD), peroxidases (PODs) and gluthatione S-transferases (GSTs) in roots ${ }^{8,30,35}$ and GSTs or glutaredoxin (GRX)/ thioredoxin (TRX) in plants ${ }^{12,13}$.

In the present study, we report results of the wholetranscriptome analysis of Solanum lycopersicum roots inoculated with $T$. harzianum T22, a PGPF widely used in several biofertilizer and biopesticide formulations ${ }^{36,37}$. The focus on this organ allowed us to provide a rootspecific model integrating the main signalling events and regulation mechanisms occurring during early root colonisation by Trichoderma, at time points (24-72 hpi) that were chosen on the basis of previous findings, to coincide with relevant stages of fungal colonisation ${ }^{29}$. Moreover, we identify, for the first time, the gene loci putatively affected by AS during the interaction with Trichoderma as well as provide evidence for a considerable number of previously unannotated AS events in tomato roots.

\section{Results}

RNA sequencing and read mapping onto $S$. lycopersicum and $T$. harzianum reference genomes

We carried out a NGS-based global transcriptomic analysis of $S$. lycopersicum roots interacting with a beneficial fungus of the rhizosphere. RNA-seq of T. harzianum T22-treated $(\mathrm{T})$ and untreated $(\mathrm{C})$ tomato roots at three time points (24, 48 and $72 \mathrm{hpi}$ ) generated a number of raw reads ranging from 46,114,872 (C72) to 57,069,524 (C24; Table 1). Approximately $13.5 \%$ of the reads were filtered out based on sequence quality, while a further $\sim 5 \%$ were discarded as a consequence of trimming operations. The percentage of high-quality reads mapped onto the tomato reference genome was $>93 \%$ for all samples (Table 1). The subset including all unmapped reads was aligned onto the T. harzianum genome. Numbers and percentages of total and uniquely mapped reads onto the fungus genome are shown in Table 1. As expected, very few reads from $C$ samples were successfully aligned. By contrast, a considerable number of uniquely mapped reads from $\mathrm{T}$ samples were identified, which increased with the time of interaction, together with 
Table 1 Sequencing and alignment statistics of the control-inoculated (C) and T. harzianum T22-inoculated (T) tomato root samples at 24,48 and $72 \mathrm{~h}$ of interaction

\begin{tabular}{|c|c|c|c|c|c|c|}
\hline \multirow[t]{2}{*}{ Sample } & \multirow[t]{2}{*}{ Raw reads (no.) } & \multirow[t]{2}{*}{ High-quality reads (no.) } & \multicolumn{2}{|c|}{$\begin{array}{l}\text { Reads aligned onto the } S \text {. lycopersicum } \\
\text { genome (no.) }\end{array}$} & \multicolumn{2}{|c|}{$\begin{array}{l}\text { Reads aligned onto the } T \text {. harzianum } \\
\text { genome (no.) }\end{array}$} \\
\hline & & & Total mapped & Uniquely mapped & Total mapped & Uniquely mapped \\
\hline $\mathrm{C} 24$ & $57,069,524$ & $46,879,826$ & $44,354,968$ (94.6\%) & $43,190,733(97.4 \%)$ & $3(-)$ & - \\
\hline $\mathrm{C} 48$ & $49,480,290$ & $40,703,519$ & $38,059,461(93.5 \%)$ & $37,101,879(97.5 \%)$ & $10(-)$ & - \\
\hline $\mathrm{C} 72$ & $46,114,872$ & $37,277,990$ & $35,376,200(94.9 \%)$ & $38,442,807(98.2 \%)$ & $7(-)$ & - \\
\hline $\mathrm{T} 24$ & $47,457,859$ & $38,753,059$ & $36,681,531(94.7 \%)$ & $35,789,320(97.6 \%)$ & 11,309 (0.54\%) & $11,299(\sim 100 \%)$ \\
\hline T48 & $50,908,596$ & $41,928,916$ & $39,703,689(94.7 \%)$ & $38,720,180(97.5 \%)$ & $24,014(1.05 \%)$ & $23,974(\sim 100 \%)$ \\
\hline $\mathrm{T} 72$ & $52,392,405$ & $43,442,331$ & $41,085,823$ (94.6\%) & $39,996,180(97.3 \%)$ & $32,288(1.40 \%)$ & $32,238(\sim 100 \%)$ \\
\hline
\end{tabular}

For each sample, percentages of uniquely mapped reads (onto tomato or fungus genomes) were calculated based on the corresponding 'Total mapped' values. For the alignment onto the T. harzianum genome, the subset including all unmapped reads onto the tomato genome was used as input data. The given values are the average of three biological replicates

the amount of the fungus in the tomato roots, as estimated by qRT-PCR of $T$. harzianum actin (Fig. S1). Box plots describing the distribution of read counts before and after normalisation are shown in Fig. S2. High Pearson's correlations between sequencing replicates were found, with average $r^{2}$ values ranging from 0.96 to 0.99 (Fig. S3).

\section{S. lycopersicum genes differentially expressed in T22-} treated plants: a specific plant response already after $24 \mathrm{~h}$

By comparing T vs. C samples, 1243 tomato DEGs were identified over the three time points. Only the DEGs detected by both EdgeR and DESeq were considered (Table S2). RNA-seq data on 12 genes, randomly selected between upregulated and downregulated DEGs, were validated by real time (RT)-qPCR and demonstrated good agreement between the two methods of gene expression profiling (mean Pearson's correlations coefficient: 0.85), with the exception of two genes (Solyc06g073530 and Solyc10g083230; Fig. S4).

The highest number of DEGs (938) was found at $24 \mathrm{~h}$ of interaction with T. harzianum (Fig. 1), the lowest (80) at $48 \mathrm{hpi}$. They were mainly downregulated at 24 and $72 \mathrm{hpi}$ (531 vs. 407 and 222 vs. 154, respectively), while the opposite was true at $48 \mathrm{hpi}$ (35 vs. 45) (Fig. 1 and Table S2). A gene with unknown function (Solyc01g090980) was the most induced at both $24\left(\log _{2}\right.$ FC: 8.23$)$ and $48 \mathrm{hpi}\left(\log _{2}\right.$ FC: 6.36). Sequence analysis revealed that this gene codes for a protein with the domain DUF4535 (InterPro ID: 027854), suggesting that it may be a short secreted protein. On the other hand, the most downregulated gene (Solyc10g075150, $\log _{2}$ FC: -5.33) was identified at $72 \mathrm{hpi}$ and codes for a nonspecific lipid-transfer protein (ns-LTP), which was not differentially expressed at 24 and $48 \mathrm{hpi}$.

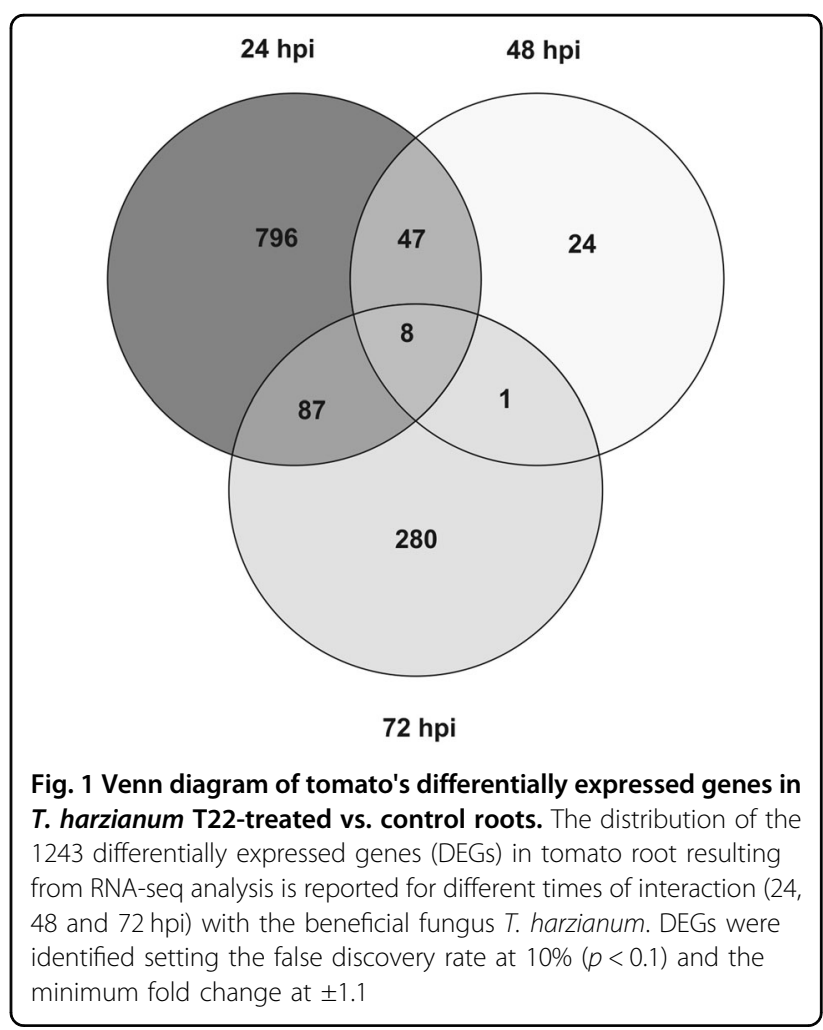

As far as Trichoderma transcripts, the complete list of DEGs and their distribution across the three time points are shown in Table S3 and Fig. S5.

According to similarities of expression trends across the three time points, the SOTA analysis grouped the 1243 tomato DEGs in five clusters (Fig. 2a, Table S2). GO analysis highlighted that in most SOTA clusters, the largest molecular function (MF) categories affected by Trichoderma treatment were 'transferase activity', followed by 


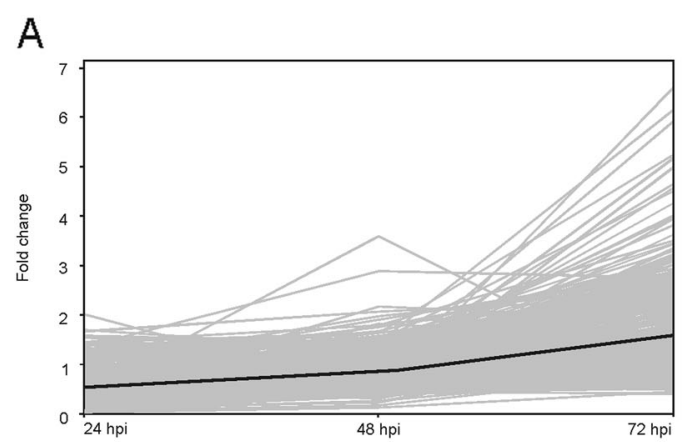

B

Cluster 1 600 genes
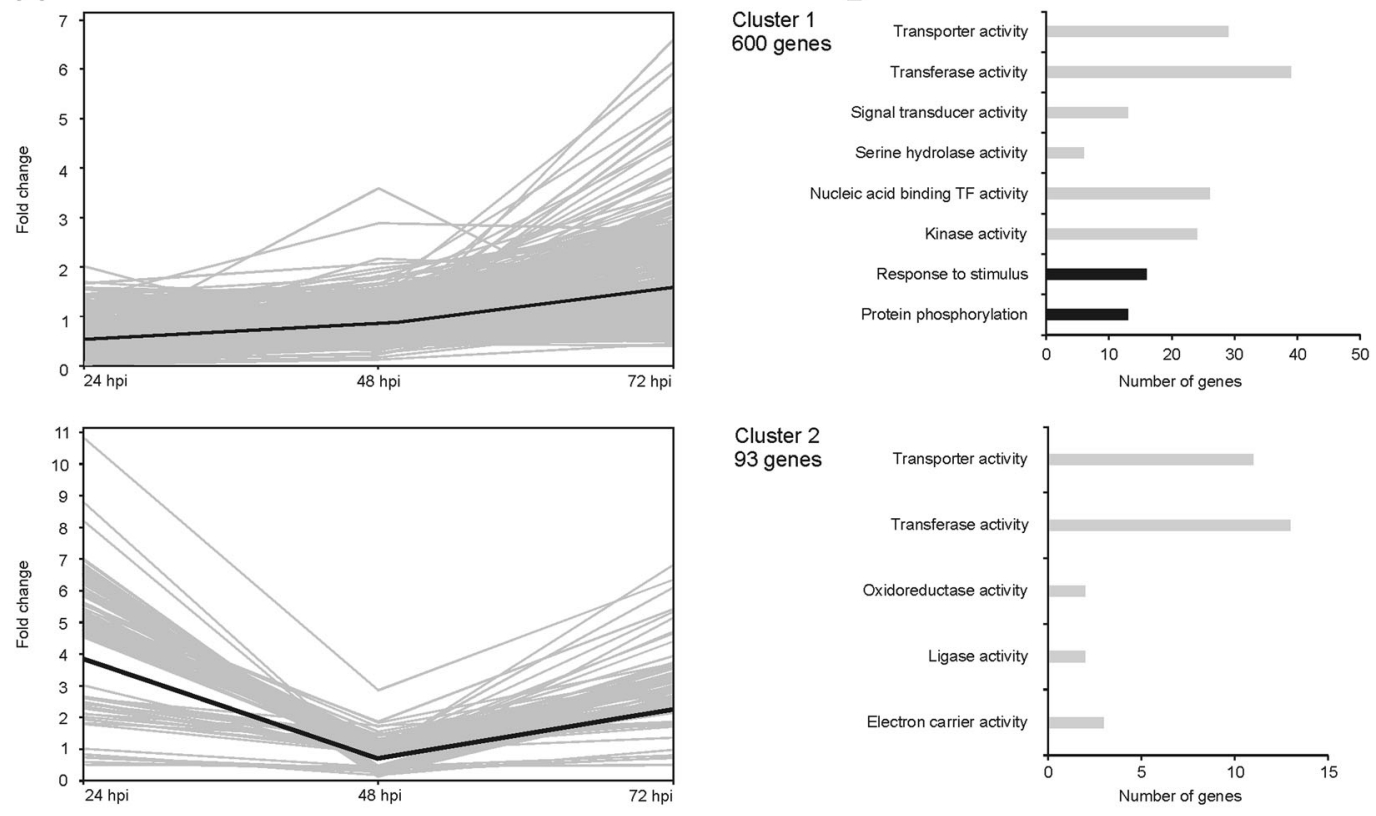

Cluster 2 93 genes
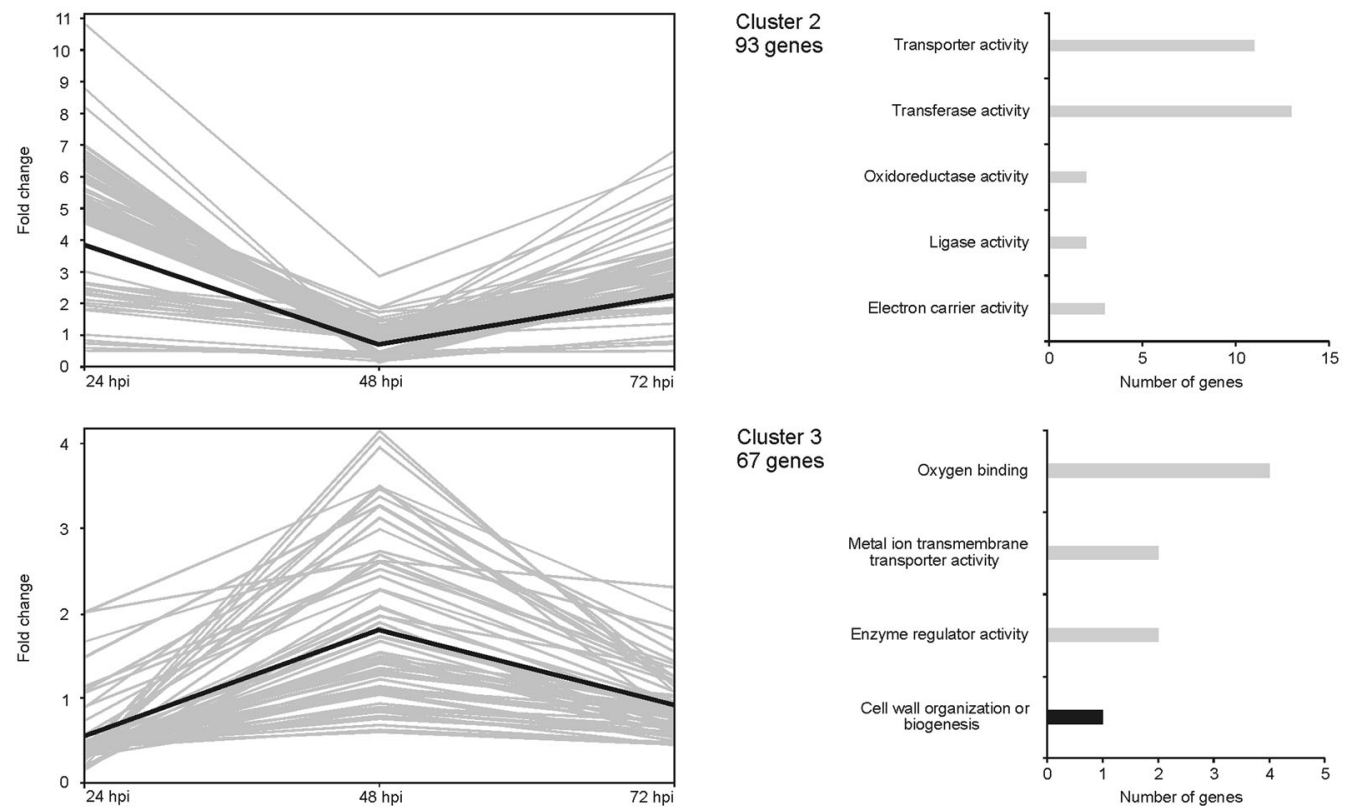

Cluster 3

67 genes
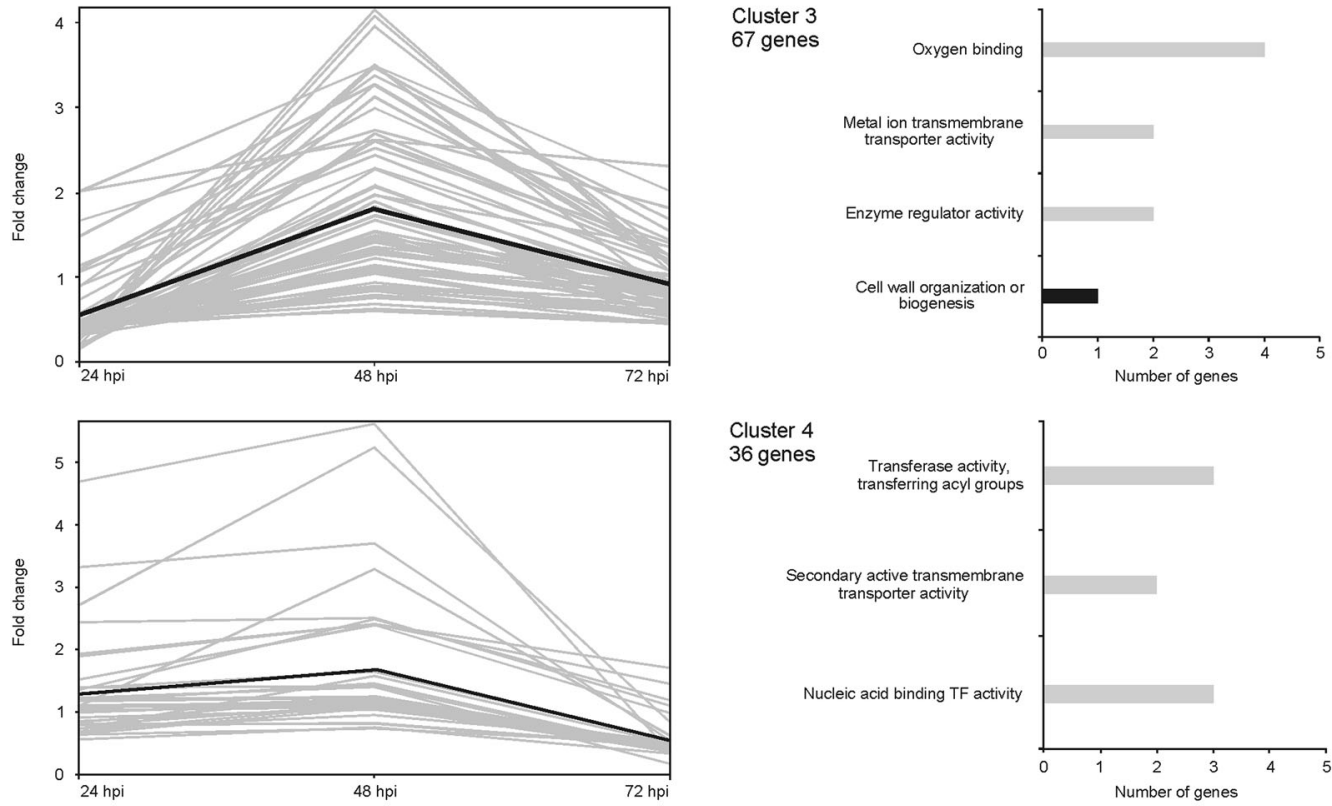

Cluster 4 36 genes
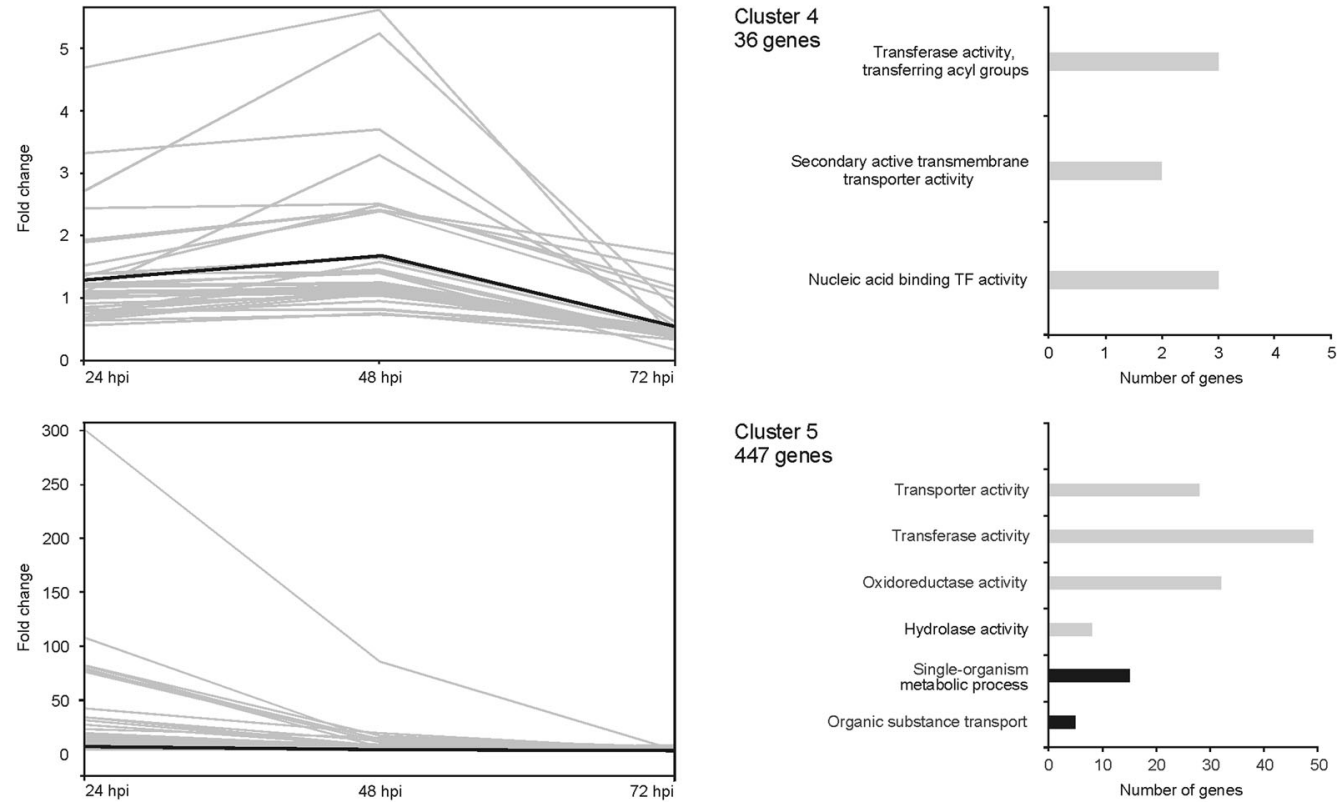

Cluster 5 447 genes

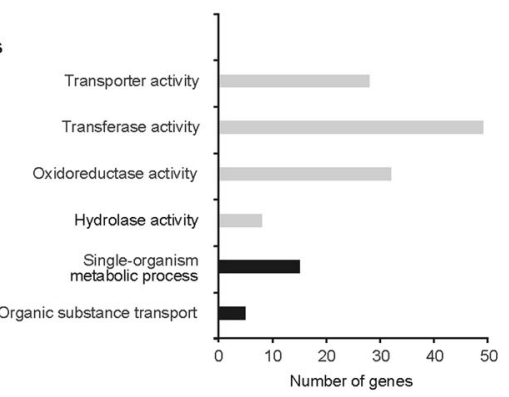

Fig. 2 (See legend on next page.) 
(see figure on previous page)

Fig. 2 Clustering of expression profiles of tomato root genes differentially expressed during the interaction with $T$. harzianum T22

and gene ontology enrichment analysis. a Grouping of tomato genes modulated by T. harzianum into five clusters, according to their expression profiles across the interaction period (24, 48 and $72 \mathrm{hpi}$ ), using the Pearson's correlation distance (SOTA method). The number of genes assigned to each cluster is indicated. The thick line indicates the cluster centroid. The $y$-axis represents the fold change of the gene expression level. b Significantly enriched gene ontology $(\mathrm{GO})$ terms associated with each expression cluster, ordered according to increasing $p$-value. Black and grey bars represent the biological process (BP) and molecular function (MF) categories, respectively. The $x$-axis represents the number of genes grouped in each GO category. TF, transcription factor

'transporter' and 'transmembrane transporter' activities (Fig. 2b, Table S4). 'Nucleic acid binding' (transcription factor) activity is one of the largest MF categories represented in cluster 1 , which groups genes that showed downregulation within the first $48 \mathrm{hpi}$ and a weak upregulation at 72 hpi. Cluster 1 includes also many genes coding for proteins with 'kinase' and 'signal transducer' activities that are possibly involved in immune response; indeed, the biological process (BP) category 'response to stimulus' is overrepresented in cluster 1 (Fig. 2b, Table S4). Furthermore, cluster 1 comprises genes classified in the 'serine hydrolase activity' MF that, similarly to the 'hydrolase activity' genes in cluster 5 , which groups genes upregulated at $24 \mathrm{hpi}$, not differentially expressed at $48 \mathrm{hpi}$ and weakly downregulated at $72 \mathrm{hpi}$, are related to protein modification. Genes having 'oxidoreductase activity' were strongly represented in cluster 5 , which groups genes upregulated at $24 \mathrm{hpi}$, not differentially expressed at $48 \mathrm{hpi}$ and weakly downregulated at $72 \mathrm{hpi}$ (Fig. 2b, Table S4).

\section{Tomato interaction with Trichoderma triggers transcriptional reprogramming of genes involved in defence, metabolism and transport}

DEGs distribution according to the MapMan ontology confirmed the modulation of tomato plant response to external stimuli, as highlighted by the over-representation of bins related to recognition, signalling and general stress response across the three time points (Fig. 3).

'Signalling' (bin 30) included DEGs mainly downregulated at 24 and $48 \mathrm{hpi}$ and upregulated at $72 \mathrm{hpi}$. DEGs associated with the Et, SA and auxin pathways were found in 'Hormone metabolism' (bin 17). Most DEGs within this bin were inhibited at 24 and 48 hpi and slightly induced at $72 \mathrm{hpi}$.

DEGs associated with general response to stimulus were classified in several bins. In particular, increased expression at $24 \mathrm{hpi}$ followed by downregulation at $72 \mathrm{hpi}$ was shown by most genes coding for cell wall-modifying enzymes (bin 10), by key genes of the phenylpropanoid pathway (bin 16) and by genes of the antioxidant enzyme machinery (bin 26). Interestingly, antioxidant and redoxsensing mechanisms were also triggered at $24 \mathrm{hpi}$ in response to Trichoderma (bin 21). On the contrary, biotic and abiotic response-associated DEGs (bin 20) showed a general repression at $24 \mathrm{hpi}$, followed by a slight induction at later time points.
At all time points, a large number of DEGs ( 26\%, 35\% and $\sim 23 \%$ at 24,48 and $72 \mathrm{hpi}$, respectively) were mapped to the category of 'Not assigned' genes (bin 35), including several genes related to general defence response. Within bin 35,155 DEGs annotated as unknown protein in iTAG were further investigated by constructing a neighbourjoining phylogenetic tree (Fig. S6). This analysis highlighted a cluster of nine sequences with a conserved gamma-thionin motif that characterise defensin proteins. Most genes coding for these putative defensins were downregulated at $24 \mathrm{hpi}$.

Transcriptomic analysis also evidenced extensive modulation of genes involved in metabolic processes during the early phases of Trichoderma root colonisation. 'Photosynthesis' (bin 1) included DEGs related to Calvin cycle or coding for structural proteins with a specific role in the photosynthetic machinery that were mainly upregulated at $24 \mathrm{hpi}$ and repressed at $72 \mathrm{hpi}(\sim 87 \%$ of down-regulated genes). 'Lipid metabolism' (bin 11) counted DEGs with roles in fatty acid and phospholipid synthesis or elongation, or coding for lipid transfer proteins (LTPs). In addition, 'Protein' (bin 29) included DEGs mostly related to post-translation modification (e.g. kinase) and protein degradation (protease/peptidase) at 24 and $72 \mathrm{hpi}$.

Several DEGs were included in 'Transport' (bin 34) over the experimental time course, being mostly upregulated within the first $48 \mathrm{~h}$. More in detail, we observed modulated expression of nutrient transport (monomers, ions and metals) and ATP-binding cassette (ABC) transporter genes.

The above responses were supported by a large recruitment of the transcriptional machinery (bin 27; Fig. 3), with 139 differentially expressed TFs over the three time points. At $24 \mathrm{hpi}, 82 \%$ of the DEGs were repressed, while at $72 \mathrm{hpi}$ an even distribution between upregulated and downregulated genes was observed. Numerous MYB (24), ERF (15) and WRKY (13) genes were identified, although other TF classes (bHLH and zinc finger family factors) were also represented.

\section{Trichoderma induces modifications in tomato transcriptional and post-transcriptional regulation}

To provide further insights into gene expression and regulatory control in the plant root-Trichoderma interaction, we analysed epigenetic, namely DNA methylation, and post-transcriptional, namely AS, modifications. 


Photosynthesis (bin 1)
Cell wall (bin 10)
Lipid metabolism (bin 11)
Secondary metabolism (bin 16)
Hormone metabolism (bin 17)
Stress (bin 20)
Redox (bin 21)
Miscellaneous (bin 26)
RNA (bin 27)
Protein (bin 29)
Signalling (bin 30)
Development (bin 33)
Transport(bin 34)
Notassigned (bin 35)
Fig. 3 MapMan analysis of tomato root genes differentially expressed during the interaction with T. harzianum T22. Organisation of tomato
root's differentially expressed genes (DEGs) in functional categories (bins) according to the MapMan ontology across the interaction period (24, 48
and 72 hpi). Genes significantly upregulated and downregulated in treated vs. control plants are indicated in red and blue, respectively. The colour
set scale is on top right corner

Interrogation of the Trichoderma-induced tomato root DEGs for epiregulators highlighted the modulation of five histone acetyltransferases (HATs) of the GNAT superfamily (known as HAG: histone acetyltransferase GCN5) and of components of the RNA-directed DNA methylation complex (Table 2). Transcription of SIHAG17 (Solyc08g068280) was activated after $24 \mathrm{hpi}$ and repressed at 72 hpi. SIHAG8, SIHAG15 and SIHAG18 (Solyc03g116860, Solyc00g272810 and Solyc12g096840) were suppressed at a very early stage of interaction and resulted as not active thereafter; by contrast, SIHAG20 (Solyc08g068710) and SIJMJ5 (Solyc08g076390, similar to histone demethylase (HDM) of the Jumonji family) were upregulated not earlier than $72 \mathrm{hpi}$. We also found that the genes coding for RNA-directed DNA methylation 1 (SIRdM1, Solyc09g082480), a component of the RNAdirected DNA methylation effector complex, and for Argonaute (AGO) slicer protein (SIAGO4-like, Solyc06g073530) were differentially expressed. In particular, the former gradually decreased its expression across the three time points $\left(\log _{2}\right.$ FC: 6.3, 3.7 and 1.8, respectively), the latter was highly suppressed at $24 \mathrm{hpi}$ and finally evened the control expression level $\left(\log _{2} \mathrm{FC}:-5.2\right.$, -2.9 , not differentially expressed). To verify whether the gene expression differences found for the RdDM players were also accompanied by cytosine methylation changes, we measured the global DNA methylation levels (in terms of percent content of 5-methylCytosine (\% 5-mC)) in the $\mathrm{C}$ and $\mathrm{T}$ samples at each time point (Fig. 4). Our results showed that control plants have no differences in methylation levels at the three time points; on the contrary in treated roots, we found a decrease in DNA methylation at $24 \mathrm{hpi}$ and an increase at $72 \mathrm{hpi}(p<0.05)$.
Table 2 Transcript abundance, expressed in $\log _{2} F C$, of five histone acetyltransferases GCN5 (SIHAG8, SIHAG15, SIHAG17, SIHAG18 and SIHAG20), a histone demethylase (HDM) of the Jumonji family (SIJMJ5), a RNA-directed DNA methylation 1 gene (SIRdM1) and a Argonaute slicer (SIAGO4-like) observed in tomato roots after 24,48 and 72 h of interaction with $T$. harzianum $\mathrm{T} 22$

\begin{tabular}{lllll}
\hline \multirow{2}{*}{ Gene abbreviation } & iTAG 2.4 ID & \multicolumn{2}{l}{ Log $_{\mathbf{2}} \mathrm{FC}$} & \\
\cline { 3 - 5 } & & $\mathbf{2 4 \mathbf { h p i }}$ & $\mathbf{4 8} \mathbf{~ h p i}$ & $\mathbf{7 2} \mathbf{~ h p i}$ \\
\hline SIHAG8 & Solyc03g116860 & -1.69 & -0.39 & -0.13 \\
SIHAG15 & Solyc00g272810 & -1.25 & -0.93 & -1.02 \\
SIHAG17 & Solyc08g068280 & 2.37 & -0.14 & -1.63 \\
SIHAG18 & Solyc12g096840 & -2.10 & -0.72 & 0.92 \\
SIHAG20 & Solyc08g068710 & 0.40 & 0.54 & 1.25 \\
SIJMJ5 & Solyc08g076390 & 0.14 & 0.34 & 1.23 \\
SIRDM1 & Solyc09g082480 & 6.30 & 3.75 & 1.84 \\
SIAG04-LIKE & Solyc06g073530 & -5.24 & -2.99 & -1.17 \\
\hline
\end{tabular}

For each gene, the iTAG (version 2.4) ID is reported. FC values below the \pm 1.1 threshold or not satisfying the FDR cutoffs are shown in italic

No statistically significant differences were found in treated roots at $48 \mathrm{hpi}$ post treatment.

The role of post-transcriptional gene regulation through AS in tomato response to Trichoderma was assessed using RSAP analysis. No differences between $\mathrm{C}$ and $\mathrm{T}$ samples at each time point were observed in terms of reads belonging to different R-SAP categories. On an average, 31\% of the reads are indicative of putative alternative transcriptional events 
(R-SAP categories: 'intron only', 'multiple annotation' and 'internal exon extension'). While the number of genes affected by splicing events was similar in all samples (Fig. 5a, b), the list of genes varied between $\mathrm{C}$ and $\mathrm{T}$ samples at the three time points. GO-enrichment analysis of genes affected by $\mathrm{AS}$ and specific for $\mathrm{C}$ or $\mathrm{T}$ samples revealed significant over-representation at $24 \mathrm{hpi}$ of the BP term 'response to stimulus', in both T. harzianum-treated and control roots.
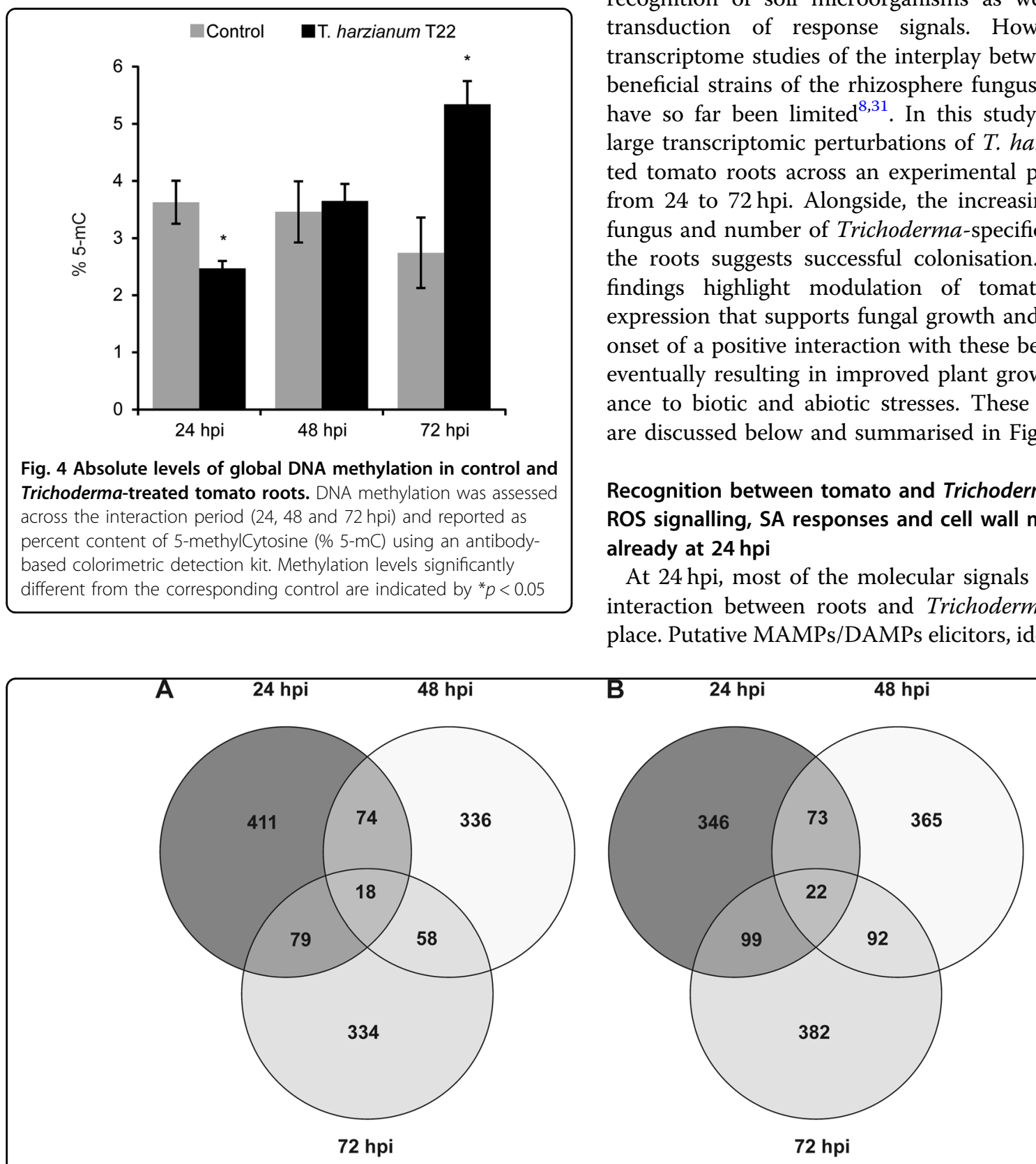

Fig. 5 Venn diagrams of tomato gene loci affected by alternative splicing in control and Trichoderma-treated roots. Distribution of novel transcribed isoforms and/or gene features ('intron only', 'multiple annotation' and 'internal exon extension' categories) resulting from comparison with iTAG2.4 annotations for $\mathbf{a}$ control and $\mathbf{b}$ Trichoderma-treated root samples at 24, 48 and 72 hpi. R-SAP analysis was run with IdentityCutoff $=95$ and CovCutoff $=98$ 

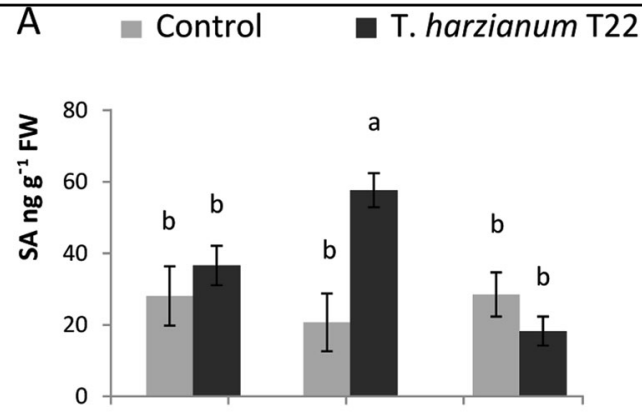

B

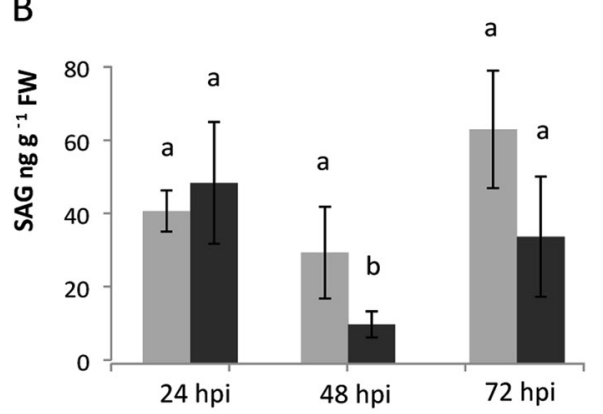

Fig. 6 Overview of modulated gene expression in tomato roots during T. harzianum T22 colonisation. The illustration is based on annotation of the DEGs in iTAG2.4 and assignment to specific

functional categories by MapMan ontology. Tomato genes that were significantly upregulated and downregulated in response to $T$.

harzianum at each time point $(24,48$ and $72 \mathrm{hpi})$ are in red and green, respectively. Gene families including both downregulated and upregulated members are in blue. Trichoderma transcripts involved in putative MAMP/DAMPs elicitation are also indicated. Solid and dashed lines represent established and hypothesised activities, respectively

trascriptome as produced by $T$. harzianum T22 (including cyclophilins, CFEM domain proteins, hydrophobins, MnPOD and glycoside hydrolases) or derived from structural modifications of the plant cell wall (polygalacturonases, PGs; xyloglucan endo-transglucosylase/hydrolase, XTHs; glycoside hydrolases, GH; laccases) (Fig. 6), were apparently recognised by root receptors, as indicated by persistent upregulation of genes encoding membranelocalised LRR proteins, cysteine-rich receptor kinases (CRKs) and by activation of a wall-associated kinase (WAK) at $72 \mathrm{hpi}$. As a hallmark of successful recognition, we observed increased transcription of tomato respiratory burst oxidase homologues (RBOHs; Fig. 6), which impinges on ROS production ${ }^{38,39}$. Expectedly, ROS accumulation drove a strong induction of the tomato root antioxidant machinery, confirmed by the activation of several related genes, such as GSTs, TRXs and PODs. These findings are consistent with the data by SalasMarina et al. ${ }^{30}$ and Brotman et al. ${ }^{8}$ in Arabidopsis after early colonisation by $T$. atroviride or $T$. asperelloides, respectively. Enhanced expression of genes coding for $\mathrm{ABC}$ membrane transporters at 24 and $48 \mathrm{hpi}$ and for components of calcium-mediated or calmodulinmediated signalling at 24 and $72 \mathrm{hpi} \mathrm{(Fig.} \mathrm{6)} \mathrm{may} \mathrm{concur}$ in the root-Trichoderma cross-talk ${ }^{16,40}$.

Several studies have indicated the importance of SA, JA and Et regulation for the response of the aerial part of the plant to Trichoderma $a^{4,13,41}$. However, the role of specific hormones has not been clarified yet, and seems to be dependent on the experimental conditions and organisms involved $^{17}$. Moreover, their role in the roots has been addressed by fewer studies ${ }^{8,31}$. In our experimental system, increased expression of SA biosynthesis and modification (phenylalanine ammonia lyase, Pal1-like and SA-dependent carboxyl methyltransferase, SAMT) and SA-responsive (e.g. pathogenesis-related, PR genes) genes suggests SA accumulation. This in turn may be responsible for the observed transcriptional repression of JA biosynthesis (allele oxide synthase, AOS; lipoxygenases, Lox 1 and Lox5, oxo-phytodienoic acid reductase 2, OPR2) and JA-responsive genes (e.g. WRKY2, defensins) across the experimental time points (Fig. 6). In addition, differential expression of SlWRKY2, SlWRKY23 and SlWRKY27, with sequence similarity with $A$. thaliana TFs (AtWRKY40, AtWRKY22 and AtWRKY41) implicated in JA-signalling and SA/JA cross-talk ${ }^{8,42,43}$, further indicates negative regulation of JA-mediated responses. In line with the enhanced expression of SA-related genes, we observed early SA increase in Trichoderma-treated tomato roots, with a significant almost threefold accumulation over the respective control at $48 \mathrm{hpi}$ ( $57.6 \mathrm{vs.}$ $20.7 \mathrm{ng} \mathrm{g}^{-1}$ FW; Fig. 7a), in contrast with no SA accumulation reported for cucumber roots inoculated with Trichodema asperellum ${ }^{44}$. The detected increase in SA was paralleled by a similar decrease of salicylic acid glucoside (SAG), mainly at $48 \mathrm{hpi} \mathrm{(10.7} \mathrm{vs.} 30.7 \mathrm{ng} \mathrm{g}^{-1} \mathrm{FW}$; Fig. 7b), consistently with the suggested role of SAG as an inactive storage form, which needs to be converted to SA to induce SA-related defences ${ }^{45}$. The temporary induction of SA confirms a possible role in avoiding excessive Trichoderma penetration within the roots ${ }^{32}$. Our analyses could not detect measurable amounts of other phytohormones, such as JA, JA-Isoleucine or the JA precursor 12-oxo-phytodienoic acid, and can suggest that jasmonate signalling is not induced in roots by Trichoderma colonisation. In agreement with our results, Arabidopsis and cucumber root interplay with Trichoderma involved increased expression of Pal1 at $24 \mathrm{hpi}$ and transient overexpression of $\operatorname{Lox} 1^{8,30,44}$. JA synthesis and JAmediated defences were suppressed also in oil palm roots at 3-12 weeks post inoculation with $T$. harzia$n_{u m^{31}}$. Et signalling appears also strongly suppressed at $24 \mathrm{hpi}$, as indicated by the downregulation of genes related to its synthesis (i.e. aminocyclopropane-1-carboxylic acid synthase, ACS and 1-aminocyclopropane-1carboxylic acid oxidase, ACO) and response (i.e. Et- 


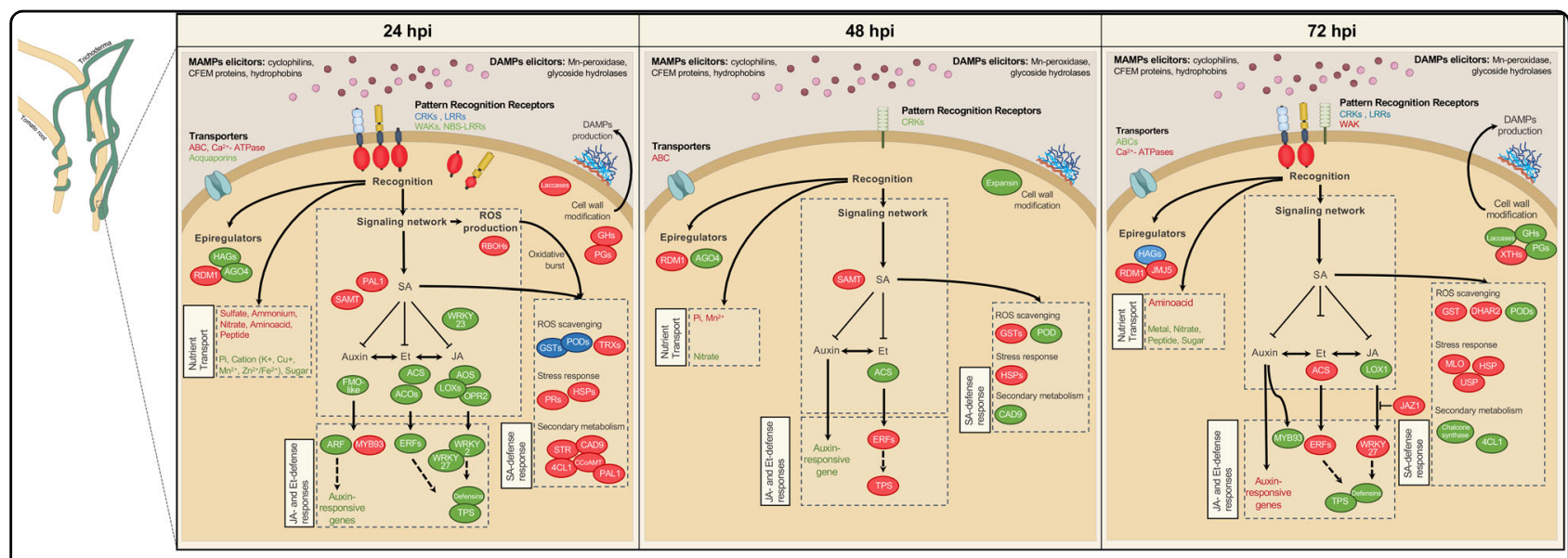

Fig. 7 Levels of salicylic acid (SA) and salicylic acid glucoside (SAG) in tomato roots. The levels of $\mathbf{a}$ SA and $\mathbf{b}$ SAG were determined in control and T. harzianum-treated roots across the interaction period ( 24,48 and $72 \mathrm{hpi}$ ) by quantitative HPLC-MS/MS. Bars represent the means \pm SD of three biological replicates. Data were subjected to analysis of variance and tested for significance $(p<0.05)$ using the Tukey's test. FW, fresh weight

responsive factors, ERFs) (Fig. 6). Notably, sequence similarity with $A$. thaliana did not identify tomato DEGs related to AtMYB72, a root-specific TF that works upstream of JA and ET in ISR signalling induced in Arabidopsis by Trichoderma ${ }^{8,46,47}$. This result may be due to a different hormonal signalling occurring in tomato, and/or due to the recruitment of a so-far unidentified tomato transcriptional regulator lacking extensive sequence similarity with AtMYB72. Given the synergistic action between Et and auxin in the roots ${ }^{48}$, suppression of Et signalling was also suggested by the downregulation of key auxin biosynthetic (flavin monooxygenases, FMOlike) and responsive (e.g. auxin response factor, ARF) genes at $24 \mathrm{hpi}$.

As a result of the above signalling network, the plant immune response was overall transiently repressed at 24 hpi, as indicated by the downregulation of most genes within the enriched BP category 'response to stimulus' (Fig. 2b). Similarly, the MapMan bins 'hormone metabolism', 'stress' and 'signalling' showed overall downregulation at $24 \mathrm{hpi}$ and upregulation at $72 \mathrm{hpi}$ (Fig. 3). This response included the downregulation of Et/JAresponsive defence genes, such as defensins and terpene synthase (TPS) (Fig. 6) and complies with the strategy adopted by the plant to allow root colonisation by ISRinducing microbes ${ }^{6,8}$. However, we observed also the upregulation of genes related to ROS detoxification (see above), defence (PR1b1; heat shock proteins, HSPs; and strictosidine synthase, STR) and cell wall strengthening (e.g. 4-coumarate:CoA ligase, 4CL; cinnamyl alcohol dehydrogenase, CAD; caffeoyl Co-A transferase, CCoAMT) (Fig. 6). These activated defences are mainly SA dependent and may be recruited to limit fungal spread within the roots, as was reported for the SA-impaired sid2 A. thaliana mutant interacting with $T$. harzianum ${ }^{32,33}$.
Tomato root modulates its transcriptional machinery to further promote a positive interaction with Trichoderma

At later times of interaction $(48-72 \mathrm{hpi})$, the observed root transcriptional response may be related to the known beneficial effects of Trichoderma in terms of plant protection (ISR priming and abiotic stress protection) and growth promotion ${ }^{7,9,49}$. In particular, the very low number of detected DEGs (80) seems to indicate $48 \mathrm{hpi}$ as a transition point, in which the plant reprograms its transcriptional machinery mainly towards oxido-reduction (GSTs) and defence (HSPs, TPS) processes (Fig. 6). At 72 hpi, changes in SA-induced defences (e.g. dehydroascorbate reductase 2, DHAR2; GSTs; HSPs; Mildew Locus $\mathrm{O}, M L O$; universal stress protein, USP) may contribute to hinder further Trichoderma penetration, but also possibly indicate induced priming. Consistent with our findings, expression of defence and ROS detoxification-related genes was systemically activated in several plant species during early interaction with Trichoderma spp. ${ }^{11,13,14,35}$ and was also stimulated during long-term interaction, taking part in the primed response to pathogens ${ }^{4,14,31}$.

Et and auxin signalling and altered redox balance can affect root architecture and stimulate root hairs ${ }^{50}$. This is also suggested by modulation of SIMYB93, similar to an auxin-induced negative regulator of lateral root development $(A t M Y B 93)^{51}$. Modified root system together with ameliorated nutrient status suggested by modulation of nutrient transporters genes (Fig. 6) can improve plant growth. Trichoderma-induced hormone signalling and ROS scavenging may further contribute to plant growth through alleviation of environmental constraints ${ }^{13,35}$. Moreover, the observed increased expression of several HSP chaperone genes may be indicative of active protein synthesis, as previously suggested ${ }^{12,14,52}$. 


\section{Perturbation of epiregulators expression, global DNA methylation and selective alterations of AS as emerging mechanisms modulating the Trichoderma-root mutualistic interaction}

Recent studies have extended our understanding of the plant epigenetic control of pathogenesis and symbio$\operatorname{sis}^{21,53,54}$. Histone modifications and DNA methylation have emerged as critical regulators of defence priming, since they affect the transcription of defence-related genes through evolutionarily highly conserved functions ${ }^{55}$. Here we identified eight epigenetic modifiers as differentially expressed in treated roots, such as HAGs, AGO and RDM1. Moreover, we found an initial hypomethylation at 24 hpi followed by recovery and hypermethylation at 48 and $72 \mathrm{hpi}$, respectively. Consistently, independent studies found not only DNA demethylation at early stages of interaction with symbiotic microorganisms ${ }^{56}$, but also interplay between different histone modifications, which has been suggested to prime a part of SAR defence genes via mechanisms that are still poorly understood ${ }^{53,54,57,58}$. Overall, based on our data, it is tempting to speculate that the observed epiregulators expression changes mediate plant protection mechanisms primed by the interaction with Trichoderma and/or other Trichoderma-induced effects. Further studies with high-throughput capabilities for detecting unknown epigenetic changes are necessary to corroborate this hypothesis.

In addition, selective alterations of AS indicate that the Trichoderma-plant interplay is regulated also at the posttranscriptional level. In particular, alongside a general enrichment of the 'response to stimulus' BP term in both control and Trichoderma-treated roots, confirming that defence genes are frequently affected by $\mathrm{AS}^{59}$, the plant response to Trichoderma recruited at $24 \mathrm{hpi}$ appears to involve specific AS events affecting defence gene loci (e.g. CC-NBS-LRRs, proteinase inhibitors I, major latex-like proteins). This finding evokes previous studies on plant-pathogen interactions reporting that most expressed genes are affected by AS, including plant defence genes ${ }^{20,25,60}$. Moreover, AS controls also root response to Trichoderma through modifications of transcriptional regulation, lipid and secondary metabolism.

To the best of our knowledge, our study is the first suggesting an involvement of epigenetic and posttranscriptional regulatory mechanisms during plant-Trichoderma interaction. However, further investigation is required to understand the dynamic engagement of these mechanisms during host-PGPF cross-talk.

Based on our results, we propose a putative model of the early tomato root-T. harzianum T22 interplay at the transcriptome level (Fig. 8). Plant-Trichoderma recognition appears to occur primarily through the MAMPs/ DAMPs-plant membrane receptors system, inducing
MTI responses. The main hormonal signalling is SA, mediating the host defence response to prevent spreading of fungal hyphae in the root vascular system. However, the root trascriptome reprogramming induced by the PGPF also includes suppression of both JA and Et biosynthesis and signalling, thus allowing root colonisation. At $72 \mathrm{hpi}$, increased transcription of Et and auxin signalling genes may result in alterations of root architecture (e.g. stimulation of root hairs). This process, together with modifications in nutrient transport occurring across the experimental period, is probably involved in the Trichoderma-induced plant growth stimulation. Our transcriptome analysis revealed also the activation of genes involved in ROS scavenging and plant defence throughout the observed interaction period. Both mechanisms may contribute to explain the known ability of T. harzianum to prime plant defences and alleviate the effects of abiotic stresses, although further investigation would be needed to validate this hypothesis.

This study of the interaction between tomato and $T$. harzianum contributes to a better understanding of the belowground events occurring between plants and beneficial rhizosphere fungi, though validation under natural conditions would be needed to account for the much higher environmental complexity. Our results may help to develop new strategies for crop biofertilization and bioprotection from biotic and abiotic stresses as well as to design new breeding strategies for the selection of crop varieties with improved ability to benefit from the interaction with beneficial rhizosphere fungi.

\section{Materials and methods \\ Plant and fungal material}

Tomato (S. lycopersicum cv. 'Crovarese') seeds and $T$. harzianum strain T22 were kindly provided by La Semiorto Sementi s.r.l. (Lavorate di Sarno, Italy) and the Department of Agricultural Sciences of the University of Naples Federico II (Portici, Italy), respectively. Seeds, sterilised in $2 \%$ sodium hypochlorite for $20 \mathrm{~min}$ and washed in sterile distilled water, were germinated in Petri dishes containing sterile filter paper in the dark at $25^{\circ} \mathrm{C}$. Seven-day-old spores of T. harzianum T22 cultured on potato dextrose agar (Sigma-Aldrich, St. Louis, Mo, USA) at $25^{\circ} \mathrm{C}$ in the dark were collected by washing the plates with sterile distilled water and brought to a concentration of $10^{8} \mathrm{~mL}^{-1}$.

\section{Hydroponic growth conditions}

Four-day-old tomato seedlings were transferred into hydroponic floating system. The plant nutrient solution (Colla G., personal communication) consisted, per litre, of $0.382 \mathrm{~g} \mathrm{Mg}\left(\mathrm{NO}_{3}\right)_{2} \cdot 6 \mathrm{H}_{2} \mathrm{O}, 0.812 \mathrm{~g} \mathrm{Ca}\left(\mathrm{NO}_{3}\right)_{2} \cdot 4 \mathrm{H}_{2} \mathrm{O}, 0.101 \mathrm{~g}$ $\mathrm{KNO}_{3}, \quad 0.320 \mathrm{~g} \quad \mathrm{~K}_{2} \mathrm{SO}_{4}, 0.205 \mathrm{~g} \mathrm{KH}_{2} \mathrm{PO}_{4}$ and $0.014 \mathrm{~g}$ Hidromix S (Valagro, Atessa (CH), Italy), pH 6.4. Plants were grown in a walk-in growth chamber at $24 / 21^{\circ} \mathrm{C}$ 


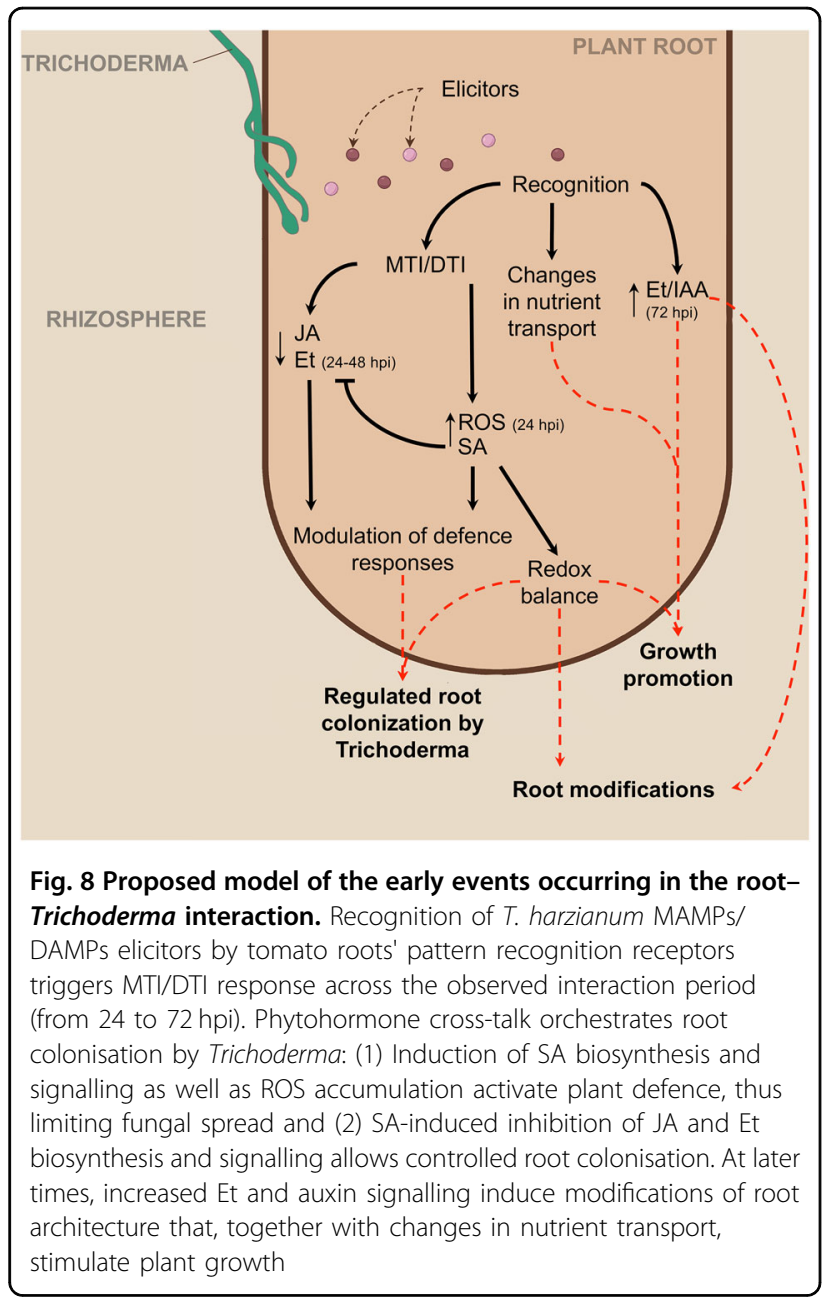

(day/night), $80 \%$ relative humidity and $16 / 8 \mathrm{~h}$ light/dark photoperiod. When tomato seedlings were 18 days old, the $T$. harzianum $\mathrm{T} 22$ inoculum was added to the nutrient solution to a final concentration of $10^{5}$ pregerminated spores $\mathrm{mL}^{-1}$. Control plants were maintained in the nutrient solution without Trichoderma. Three biological replicates, each made by pooled roots from four randomly chosen plants, were harvested for the control and Trichoderma-inoculated plants (referred to as $\mathrm{C}$ and $\mathrm{T}$ along with the paper) at 24,48 and $72 \mathrm{~h}$ post inoculation (hpi). Root samples were immediately frozen in liquid nitrogen and stored at $-80^{\circ} \mathrm{C}$ until RNA isolation.

\section{RNA isolation, CDNA library preparation and sequencing}

Total RNA was extracted from washed tomato roots (100 mg) using the RNeasy Plant Mini Kit (Qiagen, Hilden, Germany), according to the manufacturer's instructions. Complete DNA removal was obtained through oncolumn DNase I treatment using the RNase-Free DNase Set (Qiagen, Hilden, Germany). The quantity and purity of RNA was assessed using a NanoDrop ND-1000 spectrophotometer (Thermo Scientific, Wilmington, DE, USA). RNA integrity was further checked on a 2100 Bioanalyzer platform (Agilent Technologies, Santa Clara, CA, USA). Three biological replicates for each sample were used for RNA-seq. Libraries preparations and Illumina HiSeq 1500 sequencing service were carried out by Genomix4life S.r.l. (Baronissi, Italy, http://www. genomix4life.com). Raw reads (FASTQ format, $100 \mathrm{bp}$ single end, Phred +33 ) were quality filtered through the FASTX-Toolkit ${ }^{61}$, and sequencing adaptors were removed through the Trimmomatic software version $0.32^{62}$. High-quality reads were aligned onto the tomato reference genome (SL2.50) using TopHat2 version 2.0.11 ${ }^{63}$. Only uniquely mapped reads were considered for downstream analysis. Reads that did not map onto the tomato genome were further aligned against the $T$. harzianum genome (v 1.0; http://genome.jgi.doe.gov/Triha1/ Triha1.download.html). The HTSeq-count tool (HTSeq0.6.1 $)^{64}$ was used to count the reads associated with each tomato or fungal gene.

\section{Differential gene expression analysis and gene ontology (GO) enrichment}

Raw read counts were subjected to inter-sample normalisation by applying the Trimmed Mean of M-values method implemented in the EdgeR Bioconductor package (version 3.8.5) ${ }^{65}$. Differentially expressed genes (DEGs) were identified comparing results from both EdgeR and DESeq packages (version 1.18.0) ${ }^{66}$, setting a false discovery rate $(\mathrm{FDR})$ of $10 \%(P<0.1)$ and a minimum $\log _{2}$ fold change (FC) of \pm 1.1 . Only DEGs called by both methods were used in downstream analysis. Differential gene expression in $T$. harzianum was estimated between the time points ( 24 vs. $48 \mathrm{hpi}, 48$ vs. $72 \mathrm{hpi}$ and 24 vs. $72 \mathrm{hpi}$ ). In this case, the EdgeR tool was used for DEG call; quality parameters (FDR and minimum FC) were fixed as previously described. Enrichment analysis of GO terms, included in the MF and BPs domains, was performed by the topGO package, version $2.18 .0^{67}$. Identified DEGs were mapped to MapMan bins for data visualisation and pathway analysis (version 3.6.0) ${ }^{68}$. To this end, the tomato MapMan ontologies (http://www.gomapman.org/export/ current/mapman/sly_SL2.40_ITAG2.3_2015-01-09_map ping.txt.tgz) were retrieved from the GOMapMan web resource $^{69}$ and imported in the MapMan tool. The Multiple Experiment Viewer v.4.9.0 was used for clustering analysis and heatmap generation ${ }^{70}$. Self-organising tree algorithm (SOTA) was used to cluster genes on the basis of their expression profiles.

\section{RNA-seq validation by quantitative RT-PCR}

RT-qPCRs were performed on 12 randomly selected genes to validate the RNA-seq data. Primer design was carried out using the PrimerQuest tool (http://eu.idtdna. 
com/Primerquest/Home/Index). Primer sequences are listed in Table S1. The same RNA samples used for sequencing were also employed for PCR-based expression analysis. DNase-treated total RNA $(1 \mu \mathrm{g})$ was reverse transcribed using SuperScript II Reverse Transcriptase (Life Technologies, Carlsbad, CA, USA) following the protocol previously described in Tucci et al. ${ }^{4}$. Each PCR reaction consisted of $5 \mu \mathrm{L}$ of 1:20 diluted cDNA, $6.25 \mu \mathrm{L}$ of $2 \mathrm{X} \mathrm{SYBR}^{\circ}$ Select Master Mix (Life Technologies, Carlsbad, CA, USA) and $0.2 \mu \mathrm{M}$ of each gene-specific primer in a total volume of $12.5 \mu \mathrm{L}$. qRT-PCRs were performed using a 7900HT fast RT-PCR System (Applied Biosystems, Foster City, CA, USA). PCR cycling conditions were $10 \mathrm{~min}$ at $95^{\circ} \mathrm{C}(1 \mathrm{cycle})$, followed by 40 cycles of two steps at $95^{\circ} \mathrm{C}$ for $15 \mathrm{~s}$ and $60^{\circ} \mathrm{C}$ for $1 \mathrm{~min}$. Melting curves $\left(60-95^{\circ} \mathrm{C}\right)$ were recorded at the end of each run in order to check the specificity of the amplification products. Three biological replicates with three technical repetitions were tested. The $\Delta \Delta \mathrm{Ct}$ method was used for relative gene expression analysis ${ }^{71}$. The elongation factor EF1- $\alpha$ gene (GenBank Acc. No. NM_001247106.2) was used as an endogenous reference gene to normalise the gene expression values. The relative expression of each gene was calculated by using the untreated control at the same time point as a calibrator. PCR efficiencies calculated by the standard curve method were $87-107 \%$ for each primer pairs with a correlation coefficient $\left(R^{2}\right)$ of 0.99 .

\section{Quantification of $T$. harzianum tomato root colonisation by qRT-PCR}

The presence of Trichoderma was quantified in the control and T22-treated plants at 24, 48 and 72 hpi. Firststrand cDNA synthesis from root RNA and qRT-PCRs were conducted using the conditions described above, except for cDNA that was diluted 1:40 for the tomato EF1- $\alpha$ gene and 1:10 for the T. harzianum beta actin gene $^{72}$, respectively. The EF1- $\alpha$ gene was used as an endogenous reference gene to normalise the gene expression values. The relative expression of $T$. harzianum actin was calculated by using the untreated control at $24 \mathrm{hpi}$ as a calibrator.

\section{Determination of global DNA methylation}

Total DNA was isolated from the same plant materials used for RNA-seq analysis. The DNeasy Plant Mini Kit (Qiagen, Dusseldorf, Germany) was used following the manufacturer's protocol. Quantity and quality of the isolated DNA were measured using the NanoDrop ND-1000 spectrophotometer (Thermo Scientific, Wilmington, DE, USA) and Qubit 2.0 fluorometer (Life Technologies, Carlsbad, CA). For each sample, the absolute level of global DNA methylation was assessed in percentages of 5methylCytosine (\% 5 -mC) using the MethylFlash
Methylated DNA Quantification Kit (Epigentek, New York, NY). The amount of methylated DNA (proportional to the OD intensity measured at $450 \mathrm{~nm}$ ) was measured using the microplate reader spectrophotometer Multiskan FC (Thermo Scientific, Wilmington, DE, USA). \% 5-mC (methylated polynucleotide containing 50\% of 5-methylCytosine) was calculated using the formula described in the kit manual. Biological replicates were analysed in triplicate. The $t$-test was applied to discriminate amongst data averages at $95 \%$ confidence level $(p \leq 0.05)$ using the Visual Basic for Applications macro programme of the Microsoft Office Excel 2007 package.

\section{Analysis of alternative transcripts}

R-SAP (RNA-Seq Analysis Pipeline) ${ }^{73}$ was used to compare the read-to-reference genome alignments with all tomato gene tracks, as annotated in the file ITAG2.4_gene_models.gff3 downloaded from the SGN ftp server (ftp://ftp.solgenomics.net/genomes/Solanum_lycopersicu m/annotation/ITAG2.4_release/) in order to detect the novel transcribed isoforms and/or identify novel gene features. We firstly converted the tomato annotation gene tracks from the GFF3 to the GTF format using the gffread programme that comes with Cufflinks ${ }^{74}$. Then, we convert each SAM/BAM file into the PSLX format. We performed multiple R-SAP runs (one per Illumina read dataset) with IdentityCutoff $=95$ and CovCutoff $=98$. Finally all the results were collapsed into a unique data report for downstream analysis. AgriGO v2.0 ${ }^{75}$ was used to perform singular enrichment analysis (SEA) on genes affected by AS and specific for control and treated samples. $\mathrm{REVIGO}^{76}$, with the parameter 'allowed similarity' set to 'small(0.5)', was used to reduce redundancy and produce a summary of the enriched GO terms.

\section{Detection and quantification of plant hormones}

Analysis and quantification of SA, SAG, jasmonic acid (JA) and JA-Ile conjugate was carried out by high liquid chromatography-tandem mass spectrometry (HPLC-MS/MS) analysis, according to Schenk et al. ${ }^{77}$ with some modifications. Finely ground root material was extracted twice with $1 \mathrm{~mL}$ of methanol by ultrasoundassisted solid-liquid extraction (UA-SLE) for $30 \mathrm{~min}$ and centrifuged at $14,000 \mathrm{rpm}$ for $2 \mathrm{~min}$. The supernatants were collected and evaporated in a speed-vac at $30^{\circ} \mathrm{C}$ and redissolved in $250 \mu \mathrm{L}$ methanol/water 4:6, v/v. Quantitative HPLC-MS/MS analysis was performed using a Shimadzu Nexera X2 UHPLC system (Shimadzu, Milano, Italy) coupled to an Q-TRAP-6500 mass spectrometer (AB Sciex, Milan, Italy) operating in negative ion mode. The extracts were separated on a Kinetex C18 column (100 $\times$ $2.1 \mathrm{~mm}, 2.7 \mu \mathrm{m}$; Phenomenex, Bologna, Italy) using a gradient elution of $\mathrm{H}_{2} \mathrm{O}$ (A) and acetonitrile (B), both containing formic acid $0.05 \%, \mathrm{v} / \mathrm{v}$. After injection $(10 \mu \mathrm{L})$, 
the analytes were eluted using the following gradient: 0-5 min, 2\% B, 5-12 min, 2-25\% B, 12-16 min, 25-98\% B. The column was kept at $30^{\circ} \mathrm{C}$ and the flow rate was set at $0.4 \mathrm{~mL} \mathrm{~min}^{-1}$ for all chromatographic runs. At the end of each run, the column was washed with $98 \% \mathrm{~B}(5 \mathrm{~min})$ and re-equilibrated with $2 \% \mathrm{~B}(5 \mathrm{~min})$. In order to improve the analyte ionisation and to select the multiple reaction monitoring (MRM) transition, tune optimisation was carried out by the direct infusion of SA standard solution at a concentration of $2 \mu \mathrm{g} \mathrm{mL} L^{-1}$. The optimal instrument parameters were as follow: ion spray voltage (IS) $-4500 \mathrm{~V}$, source temperature (TEM) $400^{\circ} \mathrm{C}$, dwell time was $20 \mathrm{~ms}$ for each MRM transition, nebulizer gas (GS1) 40 psi, heater gas (GS2) $40 \mathrm{psi}$, curtain gas (CUR) $35 \mathrm{psi}$ and declustering potential $[-110 \mathrm{VL}]$. For the quantification and identification of analytes, MRM was used, monitoring the precursor/product transitions: $\mathrm{m} / \mathrm{z} 136.9 \rightarrow 93.0$ (collision energy $[\mathrm{CE}]-20 \mathrm{~V})$ and $136.9 \rightarrow 65.0(\mathrm{CE}-36 \mathrm{~V})$ for SA; $m / z 209.1 \rightarrow 59.0(\mathrm{CE}-25 \mathrm{~V}$ ) for JA; $m / z 290.1 \rightarrow$ 165.1 (CE $-24 \mathrm{~V}$ ) for 12-oxo-phytodienoic acid; $\mathrm{m} / \mathrm{z}$ $322.2 \rightarrow 130.1(\mathrm{CE}-30 \mathrm{~V})$ for JA-Ile conjugate and $\mathrm{m} / z$ $299.0 \rightarrow 136.9(\mathrm{CE}-25 \mathrm{~V})$ for SAG. Both Q1 and Q3 quadrupoles were maintained at unit resolution. Analyst ${ }^{\text {tm }}$ software version 1.6 was used for mass spectrometer control and data acquisition/processing.

Calibration external standard method was used to quantify SA in root extracts using seven SA concentration levels (1-62.5 $\mathrm{ng} \mathrm{mL}^{-1}$, triplicate injections for each level). The regression curve was tested with the analysis of variance (ANOVA), and a linear model was found appropriate over the tested concentration range $(y=4276.4 x+$ 43829; $R^{2}=0.99$ ). The SA amount was finally expressed as ng per gram of fresh weight \pm deviation standard $(n=$ 3). SAG levels were expressed as SA equivalents.

\section{Data availability statements}

The raw sequences from control tomato plants can be found in the European Nucleotide Archive under the study accession number PRJEB20101 (https://www.ebi.ac. uk/ena/data/view/PRJEB20101) from sample accession number ERS1623272-ERS1623280.

The raw sequences from Trichoderma-treated tomato plants can be found in the European Nucleotide Archive under the study accession number PRJEB21256 (https:// www.ebi.ac.uk/ena/data/view/PRJEB21256).

\footnotetext{
Acknowledgements

This work was partially supported by research grants from the Italian Ministry of Education, University and Research (projects GenoPOM PON02_00395_3082360 and CL.A.N. Agri-Food CTN01_00230_240864). The Authors thank Prof. G. Colla (DAFNE Department, University of Tuscia, Viterbo) who kindly shared with us his recipe for the plant nutrient solution, Dr. G. Urciuolo and S. Cangemi for performing R-SAP analysis and Mr. G. Guarino and R. Nocerino (CNR-IBBR, Portici) for excellent technical assistance.
}

\begin{abstract}
Author details
${ }^{1}$ Institute of Biosciences and BioResources, Research Division Portici, National Research Council, 80055 Portici, Italy. ${ }^{2}$ Department of Agricultural Sciences, University of Naples Federico II, 80055 Portici, Italy. Institute for Sustainable Plant Protection, National Research Council, 80055 Portici, Italy. ${ }^{4}$ Department of Pharmacy, University of Salerno, 84084 Fisciano, Italy. ${ }^{5}$ CREA, Research Centre for Vegetable and Ornamental Crops, 84098 Pontecagnano Faiano, Italy
\end{abstract}

\section{Author contributions}

M.T. and M.D.P. conceived and designed the work; N.D.A. designed the bioinformatic work; M.D.P., M.S., C.V., R.A., M.R., T.D., A.L.P., N.D.A. and M.T. performed experiments, analysed the data and contributed sections of the manuscript; M.L. critically revised the work; M.S. wrote the first draft of the manuscript and M.D.P. and M.T. wrote the paper. All authors read, reviewed and approved the submitted version.

\section{Conflict of interest}

The authors declare that they have no conflict of interest.

\section{Publisher's note}

Springer Nature remains neutral with regard to jurisdictional claims in published maps and institutional affiliations.

Supplementary Information accompanies this paper at (https://doi.org/ 10.1038/s41438-018-0079-1).

Received: 8 February 2018 Revised: 22 June 2018 Accepted: 1 July 2018 Published online: 01 January 2019

\section{References}

1. Lopez-Bucio, J., Pelagio-Flores, R. \& Herrera-Estrella, A. Trichoderma as biostimulant: exploiting the multilevel properties of a plant beneficial fungus. Sci. Hortic. 196 109-123 (2015).

2. Rouphael, Y., Cardarelli, M., Bonini, P. \& Colla, G. Synergistic action of a microbial-based biostimulant and a plant derived-protein hydrolysate enhances lettuce tolerance to alkalinity and salinity. Front Plant Sci. 8, 131 (2017).

3. Shoresh, M., Harman, G.E. \& Mastouri, F. Induced systemic resistance and plant responses to fungal biocontrol agents. Annu. Rev. Phytopathol. 48, 21-43 (2010).

4. Tucci, M., Ruocco, M., De Masi, L., De Palma, M. \& Lorito, M. The beneficial effect of Trichoderma spp. on tomato is modulated by the plant genotype. Mol. Plant Pathol. 12, 341-354 (2011).

5. Hermosa, R., Viterbo, A., Chet, I. \& Monte, E. Plant-beneficial effects of Trichoderma and of its genes. Microbiology 158, 17-25 (2012).

6. Pieterse, C. M. et al. Induced systemic resistance by beneficial microbes. Annu. Rev. Phytopathol. 52, 347-375 (2014).

7. Martinez-Medina, A. et al. Shifting from priming of salicylic acid- to jasmonic acid-regulated defences by Trichoderma protects tomato against the root knot nematode Meloidogyne incognita. New Phytol. 213, 1363-1377 (2017).

8. Brotman, Y. et al. Trichoderma-plant root colonization: escaping early plant defense responses and activation of the antioxidant machinery for saline stress tolerance. PLoS Pathog. 9, e1003221 (2013).

9. Zhang, S. W., Gan, Y. T. \& Xu, B. L. Application of plant-growth-promoting fungi Trichoderma longibrachiatum T6 enhances tolerance of wheat to salt stress through improvement of antioxidative defense system and gene expression Front. Plant Sci. 7, 1405 (2016).

10. Fu, J., Liu, Z., Li, Z., Wang, Y. \& Yang, K. Alleviation of the effects of saline-alkaline stress on maize seedlings by regulation of active oxygen metabolism by Trichoderma asperellum. PLOS ONE 12, e0179617 (2017).

11. Alfano, G. et al. Systemic modulation of gene expression in tomato by Trichoderma hamatum 382. Phytopathology 97, 429-437 (2007).

12. Shoresh, M. \& Harman, G. E. The molecular basis of shoot responses of maize seedlings to Trichoderma harzianum T22 inoculation of the root: a proteomic approach. Plant Physiol. 147, 2147-2163 (2008). 
13. Mathys, J. et al. Genome-wide characterization of ISR induced in Arabidopsis thaliana by Trichoderma hamatum T382 against Botrytis cinerea infection. Front. Plant Sci. 3, 108 (2012)

14. De Palma, M. et al. Suppression subtractive hybridization analysis provides new insights into the tomato (Solanum lycopersicum L.) response to the plant probiotic microorganism Trichoderma longibrachiatum MK1. J. Plant Physiol. 190, 79-94 (2016).

15. Hermosa, R. et al. The contribution of Trichoderma to balancing the costs of plant growth and defense. Int Microbiol 16, 69-80 (2013).

16. Ruocco, M. et al. Multiple roles and effects of a novel Trichoderma hydrophobin. Mol. Plant-Microbe Interact. 28, 167-179 (2015).

17. Vos, C. M., De Cremer, K., Cammue, B. P. \& De Coninck, B. The toolbox of Trichoderma spp. in the biocontrol of Botrytis cinerea disease. Mol. Plant Pathol. 16, 400-412 (2015).

18. Lace, B. et al. Gate crashing arbuscular mycorrhizas: in vivo imaging shows the extensive colonization of both symbionts by Trichoderma atroviride. Environ. Microbiol. Rep. 7, 64-77 (2015).

19. Contreras-Cornejo, H. A., Macias-Rodriguez, L., Cortes-Penagos, C. \& LopezBucio, J. Trichoderma virens, a plant beneficial fungus, enhances biomass production and promotes lateral root growth through an auxin-dependent mechanism in Arabidopsis. Plant Physiol. 149, 1579-1592 (2009).

20. Howard, B. E. et al. High-throughput RNA sequencing of pseudomonasinfected Arabidopsis reveals hidden transcriptome complexity and novel splice variants. PLOS ONE 8, e74183 (2013).

21. Zogli, P. \& Libault, M. Plant response to biotic stress: Is there a common epigenetic response during plant-pathogenic and symbiotic interactions? Plant Sci. 263, 89-93 (2017).

22. Da, K., Nowak, J. \& Flinn, B. Potato cytosine methylation and gene expression changes induced by a beneficial bacterial endophyte, Burkholderia phytofirmans strain PsJN. Plant Physiol. Biochem. 50, 24-34 (2012).

23. Chamala, S., Feng, G., Chavarro, C. \& Barbazuk, W. B. Genome-wide identification of evolutionarily conserved alternative splicing events in flowering plants. Front Bioeng. Biotechnol. 3, 33 (2015).

24. Yang, S., Tang, F. \& Zhu, H. Alternative splicing in plant immunity. Int J. Mol. Sci. 15, 10424-10445 (2014)

25. Tan, G. X. et al. Transcriptome analysis of the compatible interaction of tomato with Verticillium dahliae using RNA-sequencing. Front. Plant Sci. 6, 428 (2015).

26. Ruzicka, D. et al. Inside arbuscular mycorrhizal roots - molecular probes to understand the symbiosis. Plant Genome 6, https://doi.org/10.3835/ plantgenome2012.06.0007 (2013).

27. Sun, Y. \& Xiao, H. Identification of alternative splicing events by RNA sequencing in early growth tomato fruits. BMC Genom. 16, 948 (2015).

28. Yedidia, I. I., Benhamou, N. \& Chet, I. I. Induction of defense responses in cucumber plants (Cucumis sativus L.) by the biocontrol agent Trichoderma harzianum. Appl. Environ. Microbiol. 65, 1061-1070 (1999).

29. Chacon, M. R. et al. Microscopic and transcriptome analyses of early colonization of tomato roots by Trichoderma harzianum. Int. Microbiol. 10, 19-27 (2007).

30. Salas-Marina, M. A. et al. Colonization of Arabidopsis roots by Trichoderma atroviride promotes growth and enhances systemic disease resistance through jasmonic acid/ethylene and salicylic acid pathways. Eur. J. Plant Pathol. 131, 15-26 (2011).

31. Ho, C. L. et al. De novo transcriptome analyses of host-fungal interactions in oil palm (Elaeis guineensis Jacq.). BMC Genom. 17, 66 (2016).

32. Alonso-Ramirez, A. et al. Salicylic acid prevents Trichoderma harzianum from entering the vascular system of roots. Mol. Plant Pathol. 15, 823-831 (2014).

33. Martinez-Medina, A., Appels, F. V. W. \& van Wees, S. C. M. Impact of salicylic acid- and jasmonic acid-regulated defences on root colonization by Trichoderma harzianum T-78. Plant Signal Behav. 12, e1345404 (2017).

34. Ahmad, P. et al. Role of Trichoderma harzianum in mitigating $\mathrm{NaCl}$ stress in Indian mustard (Brassica juncea L.) through antioxidative defense system. Front. Plant Sci. 6, 868 (2015)

35. Mastouri, F., Bjorkman, T. \& Harman, G. E. Trichoderma harzianum enhances antioxidant defense of tomato seedlings and resistance to water deficit. Mol. Plant Microbe Interact. 25, 1264-1271 (2012).

36. Vinale, F. et al. Trichoderma-plant-pathogen interactions. Soil Biol. Biochem. 40 $1-10$ (2008)

37. Moran-Diez, E. et al. Transcriptomic response of Arabidopsis thaliana after $24 \mathrm{~h}$ incubation with the biocontrol fungus Trichoderma harzianum. J. Plant Physiol. 169, 614-620 (2012).
38. Suzuki, N. et al. Respiratory burst oxidases: the engines of ROS signaling. Curr Opin. Plant Biol. 14, 691-699 (2011).

39. Kimura, S., Waszczak, C., Hunter, K. \& Wrzaczek, M. Bound by fate: the role of reactive oxygen species in receptor-like kinase signaling. Plant Cell 29, 638-654 (2017).

40. Navazio, L. et al. Calcium-mediated perception and defense responses activated in plant cells by metabolite mixtures secreted by the biocontrol fungus Trichoderma atroviride. BMC Plant Biol. 7, 41 (2007).

41. Contreras-Cornejo, H. A., Macias-Rodriguez, L., Beltran-Pena, E., Herrera-Estrella, A. \& Lopez-Bucio, J. Trichoderma-induced plant immunity likely involves both hormonal- and camalexin-dependent mechanisms in Arabidopsis thaliana and confers resistance against necrotrophic fungi Botrytis cinerea. Plant Signal Behav. 6, 1554-1563 (2011).

42. Asai, T. et al. MAP kinase signalling cascade in Arabidopsis innate immunity. Nature 415, 977-983 (2002)

43. Higashi, K. et al. Modulation of defense signal transduction by flagellininduced WRKY41 transcription factor in Arabidopsis thaliana. Mol. Genet. Genom. 279, 303-312 (2008)

44. Shoresh, M., Yedidia, I. \& Chet, I. Involvement of jasmonic acid/ethylene signaling pathway in the systemic resistance induced in cucumber by Trichoderma asperellum T203. Phytopathology 95, 76-84 (2005).

45. Vlot, A. C., Dempsey, D. A. \& Klessig, D. F. Salicylic acid, a multifaceted hormone to combat disease. Annu Rev. Phytopathol. 47, 177-206 (2009).

46. Van der Ent, S. et al. MYB72 is required in early signaling steps of rhizobacteriainduced systemic resistance in Arabidopsis. Plant Physiol. 146, 1293-1304 (2008).

47. Segarra, G., Van der Ent, S., Trillas, I. \& Pieterse, C. M. MYB72, a node of convergence in induced systemic resistance triggered by a fungal and a bacterial beneficial microbe. Plant Biol. (Stuttg.) 11, 90-96 (2009).

48. Ruzicka, K. et al. Ethylene regulates root growth through effects on auxin biosynthesis and transport-dependent auxin distribution. Plant Cell 19, 2197-2212 (2007)

49. Harman, G. E., Howell, C. R., Viterbo, A., Chet, I. \& Lorito, M. Trichoderma species -opportunistic, avirulent plant symbionts. Nat. Rev. Microbiol 2, 43-56 (2004).

50. Carol, R. J. \& Dolan, L. The role of reactive oxygen species in cell growth: lessons from root hairs. J. Exp. Bot. 57, 1829-1834 (2006).

51. Gibbs, D. J. et al. AtMYB93 is a novel negative regulator of lateral root development in Arabidopsis. New Phytol. 203, 1194-1207 (2014).

52. Segarra, G. et al. Proteome, salicylic acid, and jasmonic acid changes in cucumber plants inoculated with Trichoderma asperellum strain T34. Proteomics 7, 3943-3952 (2007).

53. Saijo, Y. \& Reimer-Michalski, E.-M. in Epigenetic Memony and Control in Plants (eds Gideon Grafi \& Nir Ohad) 57-76 (Springer, Berlin, Heidelberg, 2013).

54. Ding, B. \& Wang, G. L. Chromatin versus pathogens: the function of epigenetics in plant immunity. Front. Plant Sci. 6, 675 (2015).

55. Espinas, N. A., Saze, H. \& Saijo, Y. Epigenetic control of defense signaling and priming in plants. Front. Plant Sci. 7, 1201 (2016).

56. Satgé, $C$. et al. Reprogramming of DNA methylation is critical for nodule development in Medicago truncatula. Nat. Plants 2, 16166 (2016).

57. Gutzat, R. \& Mittelsten Scheid, O. Epigenetic responses to stress: triple defense? Curr. Opin. Plant Biol. 15, 568-573 (2012).

58. Conrath, U., Beckers, G. J., Langenbach, C. J. \& Jaskiewicz, M. R. Priming for enhanced defense. Annu. Rev. Phytopathol. 53, 97-119 (2015).

59. Gassmann, W. Alternative splicing in plant defense. Curr. Top. Microbiol. Immunol. 326, 219-233 (2008).

60. Shang, $X .$, Cao, Y. \& Ma, L. Alternative splicing in plant genes: a means of regulating the environmental fitness of plants. Int. J. Mol. Sci. 18, 432 (2017). https://doi.org/10.3390/ijms18020432

61. Pearson, W. R., Wood, T., Zhang, Z. \& Miller, W. Comparison of DNA sequences with protein sequences. Genomics 46, 24-36 (1997).

62. Bolger, A. M., Lohse, M. \& Usadel, B. Trimmomatic: a flexible trimmer for Illumina sequence data. Bioinformatics 30, 2114-2120 (2014).

63. Kim, D. et al. TopHat2: accurate alignment of transcriptomes in the presence of insertions, deletions and gene fusions. Genome Biol. 14, R36 (2013).

64. Anders, S., Pyl, P. T. \& Huber, W. HTSeq-a Python framework to work with highthroughput sequencing data. Bioinformatics 31, 166-169 (2015).

65. Robinson, M. D., McCarthy, D. J. \& Smyth, G. K. edgeR: a Bioconductor package for differential expression analysis of digital gene expression data. Bioinformatics 26, 139-140 (2010) 
66. Anders, S. \& Huber, W. Differential expression analysis for sequence count data. Genome Biol. 11, R106 (2010).

67. Alexa, A., Rahnenfuhrer, J. \& Lengauer, T. Improved scoring of functional groups from gene expression data by decorrelating $\mathrm{GO}$ graph structure. Bioinformatics 22, 1600-1607 (2006).

68. Thimm, O. et al. MAPMAN: a user-driven tool to display genomics data sets onto diagrams of metabolic pathways and other biological processes. Plant J. 37, 914-939 (2004).

69. Ramsak, Z. et al. GoMapMan: integration, consolidation and visualization of plant gene annotations within the MapMan ontology. Nucleic Acids Res. 42, D1167-D1175 (2014).

70. Herrero, J., Valencia, A. \& Dopazo, J. A hierarchical unsupervised growing neural network for clustering gene expression patterns. Bioinformatics $\mathbf{1 7}$ 126-136 (2001).

71. Pfaffl, M.W. A new mathematical model for relative quantification in real-time PCR. Nucleic Acids Res. 29, e45 (2001)
72. Scherm, B., Schmoll, M., Balmas, V., Kubicek, C. P. \& Migheli, Q. Identification of potential marker genes for Trichoderma harzianum strains with high antagonistic potential against Rhizoctonia solani by a rapid subtraction hybridization approach. Curr. Genet. 55, 81-91 (2009).

73. Mittal, V. K. \& McDonald, J. F. R-SAP: a multi-threading computational pipeline for the characterization of high-throughput RNA-sequencing data. Nucleic Acids Res. 40, e67 (2012)

74. Trapnell, C. et al. Transcript assembly and quantification by RNA-Seq reveals unannotated transcripts and isoform switching during cell differentiation. Nat. Biotechnol. 28, 511-515 (2010).

75. Tian, T. et al. agriGOv2.0: a GO analysis toolkit for the agricultural community, 2017 update. Nucleic Acids Res. 45, W122-W129 (2017).

76. Supek, F., Bosnjak, M., Skunca, N. \& Smuc, T. REVIGO summarizes and visualizes long lists of gene ontology terms. PLoS ONE 6, e21800 (2011).

77. Schenk, S. T. et al. N-Acyl-homoserine lactone primes plants for cell wall reinforcement and induces resistance to bacterial pathogens via the salicylic acid/oxylipin pathway. Plant Cell 26, 2708-2723 (2014). 